\title{
Brown Dwarf Binaries
}

\author{
Katelyn N. Allers \\ Department of Physics \& Astronomy, Bucknell University \\ Lewisburg, PA 17837, U.S.A \\ email: k.allers@bucknell.edu
}

\begin{abstract}
Nearly 500 brown dwarfs have been discovered in recent years. The majority of these brown dwarfs exist in the solar neighborhood, yet determining their fundamental properties (mass, age, temperature \& metallicity) has proved to be quite difficult, with current estimates relying heavily on theoretical models. Binary brown dwarfs provide a unique opportunity to empirically determine fundamental properties, which can then be used to test model predictions. In addition, the observed binary fractions, separations, mass ratios, \& orbital eccentricities can provide insight into the formation mechanism of these low-mass objects. I will review the results of various brown dwarf multiplicity studies, and will discuss what we have learned about the formation and evolution of brown dwarfs by examining their binary properties as a function of age and mass.
\end{abstract}

\section{Introduction}

In this review, I will focus on brown dwarf binaries, where the mass of the primary is less than $0.075 \mathrm{M}_{\odot}$. Many of the studies presented at this meeting are dealing with samples of thousands, or even tens of thousands of stellar binaries. In contrast, the number of known brown dwarf binaries is $\sim 55$. The low number of brown dwarf binaries can be attributed to 3 main properties of brown dwarfs: First, relative to stars, brown dwarfs are rare. The ratio of brown dwarfs to stars is $\sim 20 \%$ (Luhman et al. 2007). Second, brown dwarfs are intrinsically faint. A $0.06 \mathrm{M}_{\odot}$ brown dwarf is $\sim 100$ times fainter than a 0.10 $\mathrm{M}_{\odot}$ low-mass star. This means multiplicity studies of brown dwarfs are usually limited to

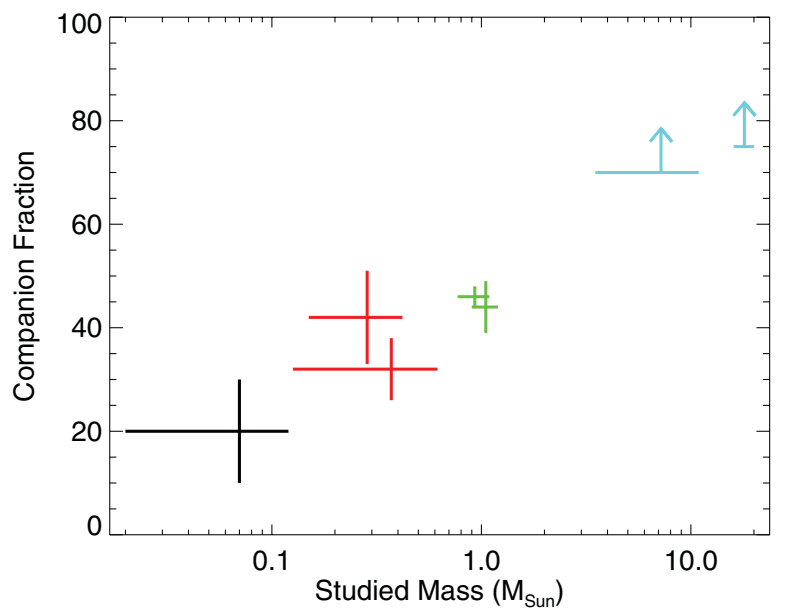

Figure 1. Companion fraction (including binaries and higher order multiple systems) as a function of primary mass. Data points from Mason et al. (2009), Kouwenhoven et al. (2007), Raghavan et al. (2010), Duquennoy \& Mayor (1991), Fischer \& Marcy (1992), Bergfors et al. (2010) and Allen (2007). 
the solar neighborhood. Third, the binary fraction of brown dwarfs is significantly lower than that of stars. Figure 1 illustrates the trend of decreasing multiplicity fraction with lower primary mass.

\section{Properties of Brown Dwarf Binaries}

Given the small number of known brown dwarf binaries, it is very important that these binaries be consistently characterized. For the following analysis, I use the list of brown dwarf binaries from Liu et al. (2010), supplemented with new binary discoveries from Artigau et al. (2011), Burgasser et al. (2011a), Burgasser et al. (2011b), Gelino et al. (2011) and Liu et al. (2011). Where possible, I use values for total masses and semimajor axes determined from orbital monitoring (Dupuy et al. 2010, Konopacky et al. 2010). Other missing orbital data were taken from www.vlmbinaries.org. Note that this sample does not include known brown dwarf binaries in star forming regions or known young moving groups (which are discussed separately). Figure 2 shows distributions of observational parameters for brown dwarfs, compared to low-mass stars and solar-mass stars. It is important to note that the multiplicity properties of brown dwarfs are an extension of mass-dependent trends.

Compared to stars, brown dwarf binaries exhibit a clear tendency toward equal mass systems, with $\sim 75 \%$ of brown dwarf binaries having $M_{2} / M_{1} \geqslant 0.8$. The lack of many low mass ratio systems could, in part, be due to the difficulty in detecting the secondary components. High resolution imaging surveys, however, are complete for $M_{2} / M_{1} \gtrsim 0.6$, so the peak in the mass ratio distribution would be unaffected by incompleteness effects at low mass ratios. Recent advances in image reduction and analysis, however, have led to the recent discovery of a few low mass ratio systems (Burgasser et al. 2011b, Liu et al. 2011, Liu et al. 2010).

The peak of the separation distribution of brown dwarfs is $\sim 3$ AU. High resolution imaging surveys are typically not sensitive to binaries with separations smaller than a few AU. As noted by Burgasser et al. (2007), the peak of the separation distribution is very close to the incompleteness limit, and thus the actual peak could lie at closer separations. Joergens (2008), however, conducted an RV search for low-mass star and brown dwarf binaries in Chamaeleon and concluded that the binary frequency for sub-AU separations

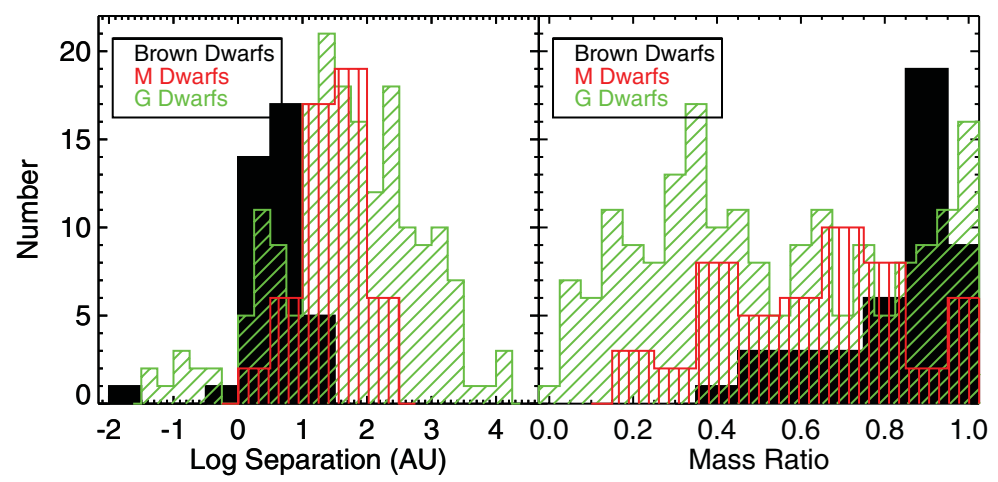

Figure 2. Comparison of binary properties for brown dwarfs and stars. Left: Histograms of projected separations for brown dwarf binaries compared to solar-type stars and low-mass stars. Right: Histograms of mass ratios (q) for binary brown dwarfs, solar-type stars and low-mass stars. Data for brown dwarf binaries is described in the text. Data for stars comes from Raghavan et al. (2010) for solar type stars and Bergfors et al. (2010) for M dwarfs. 
is small $(\lesssim 10 \%)$. Thus, the distribution of brown dwarf binary separations is likely a few AU. Perhaps a more interesting feature of the separation distribution is narrow range of separations for brown dwarf binaries. 90\% of brown dwarf binaries are more closely separated than $10 \mathrm{AU}$, in contrast to solar-type binaries which have a much broader range of separation.

\section{Young Brown Dwarf Binaries}

Because the nearest star forming regions are $\sim 125 \mathrm{pc}$ away, high resolution imaging surveys for young brown dwarf binaries, are typically only complete for separations greater than 10 AU. On the other hand, RV searches (e.g. Joergens et al. 2008) are sensitive to only small separations $(<3 \mathrm{AU})$. This means that currently, we do not have the observational capability to find 3-10 AU separation brown dwarf binaries (i.e. the peak of the field dwarf distribution) in young clusters. One solution to this problem is to search for nearby, young brown dwarf binaries in the field (e.g. Allers et al. 2010), but the low number of known young field brown dwarfs make such studies difficult.

Figure 3 compares the binary properties for "normal" field brown dwarfs and young brown dwarfs. Young brown dwarf binaries appear to have lower mass ratios and larger separations than field brown dwarfs. Both of these effects could result from observational bias and small number statistics. Still, there appears to be a handful of unusually wide separation ( $\gtrsim 500 \mathrm{AU}$ ) young brown dwarf binaries. It is important to note that these wide systems are very rare (f $\sim 1-2 \%$, Kraus \& Hillenbrand 2009), and are unlikely to survive the dispersal of their native cluster (Close et al. 2007). Biller et al. (2011) determined that the frequency of brown dwarf binaries at separations $>10 \mathrm{AU}$ is statistically the same as the wide binary frequency for field brown dwarfs. Clearly, the most important advance in the study of young brown dwarf binaries will be the ability to probe the crucial 3-10 AU separations in star forming regions with JWST or the next generation of large telescopes (e.g. GMT, TMT, ELT).

\section{Testing Formation Models}

Brown dwarfs present a particular challenge to models of star formation, as their mass is significantly below the typical Jeans mass in star forming clouds. Theories for the

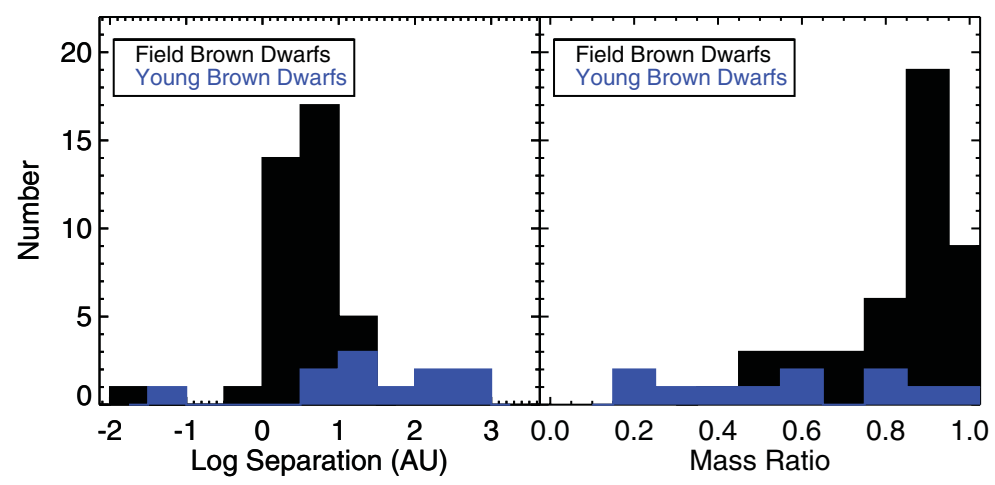

Figure 3. Comparison of binary properties for brown dwarfs in the field (age $\sim 1 \mathrm{Gyr}$ ) to young brown dwarfs in star-forming regions and moving groups (age $\lesssim 10 \mathrm{Myr}$ ). Left: Histograms of projected separations. Right: Histograms of mass ratios (q). Data for field brown dwarf binaries is described in the text. Data for young brown dwarf binaries (primary mass $<0.075 \mathrm{M}_{\odot}$ ) is taken from Table 1 of Biller et al. (2011). 
formation of brown dwarfs fall into two categories: 1) lower the Jeans mass via hierarchical or turbulent fragmentation (e.g. Bate 2009) or 2) circumvent the Jeans mass by either ejecting the brown dwarf from its embryo (e.g. Reipurth and Clarke 2001) or forming the brown dwarf from a gravitational instability in a circumstellar disk (Stamatellos \& Whitworth 2009). These different formation mechanisms would result in very different binary frequencies, binding energies, and orbital eccentricities.

The close separations and low binary fraction of field brown dwarf binaries initially seemed to support the ejection scenario for forming brown dwarfs. Burgasser et al. (2007) present binding energies for several field brown dwarfs and reported that the widest brown dwarf binaries had binding energies 10-20 times higher than field stars. Recent discoveries of less bound field brown dwarfs indicate that the binding energy lower limit for brown dwarf binaries is similar to stars (Figure 4) which argues for a common formation mechanism. Wide, young binaries having extremely low binding energies (Figure 4) could not have formed via ejection.

An additional test of formation models can be made by comparing model predictions for orbital eccentricities. Dupuy \& Liu (2011) compare the orbital eccentricities and periods of very low-mass stellar and brown dwarf binaries to the predictions of a numerical simulation of turbulent fragmentation of a cloud and gravitational instability in a massive circumstellar disk. The observed eccentricity distribution agrees quite well with the Bate (2009) model, whereas the Stamatellos \& Whitworth (2009) simulation predicts higher eccentricities than observed. As more brown binaries are discovered and well characterized, it will become increasingly important for theoretical models to provide predictions of brown dwarf binary properties.

\section{Testing Atmospheric and Evolutionary Models}

The atmospheres of brown dwarfs are very complicated, with molecules providing the dominant opacity source. Cloud formation, dust subtended in the photosphere, and non-local chemical equilibrium make brown dwarfs a particularly difficult challenge for modellers (see contributions by F. Allard and A. Burrows). The complexity of the atmospheric models manifests itself in evolutionary models as well (e.g. Burrows et al. 2011).

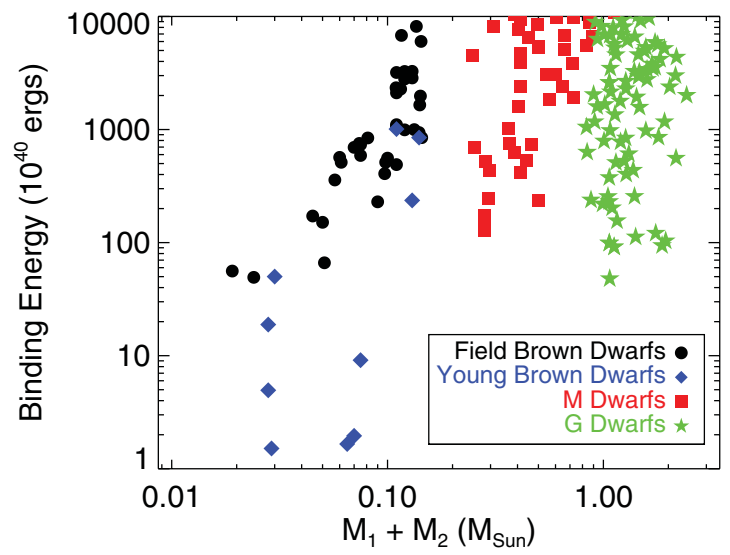

Figure 4. Binding Energies for brown dwarfs in the field (age $\sim 1$ Gyr), young brown dwarfs in star-forming regions and moving groups (age $\lesssim 10 \mathrm{Myr}$ ), low-mass stars (Bergfors et al. 2010) and solar-mass stars (Raghavan et al. 2010). 
Models atmospheres and isochrones are widely used to estimate the properties of brown dwarfs and extrasolar planets, but the reliability of these results depends on the fidelity of the models. Brown dwarf binaries provide a unique opportunity to empirically determine the fundamental properties of brown dwarfs, which can in turn be used to test the predictions of atmospheric and evolutionary models.

Orbital monitoring has allowed determination of high precision dynamical masses for a handful of brown dwarf binaries (e.g. Dupuy et al. 2011, Konopacky et al. 2010, Liu et al. 2008). One can then compare the $\log (\mathrm{g})$ and effective temperatures derived by fitting model atmospheres to the spectra of the binary with those inferred by comparing the luminosity and mass of the binary to evolutionary models. Thus far, the temperatures derived from these two methods differ by $\sim 100-300 \mathrm{~K}$ (Dupuy et al. 2009, Liu et al. 2008). Whether this discrepancy arises from the atmospheric or evolutionary models is unclear. Metallicity could also factor into this difference (Burrows et al. 2011), but a large divergence $(\gtrsim 1 \mathrm{dex})$ from solar $[\mathrm{Fe} / \mathrm{H}]$ would be required to account for a $200 \mathrm{~K}$ difference in effective temperature.

If the age and/or metallicity of the brown dwarf is independently determined, one can compare the measured luminosity to the luminosity inferred by evolutionary models. To date, the only brown dwarf binary able to provide this test of the models is HD130948BC, which has a known age and metallicity from its G2V primary star (Dupuy et al. 2009). In this particular case, the luminosity predicted by the evolutionary models is $\sim 2$ times lower than the measured value. Current orbital monitoring surveys should produce dynamical masses for additional brown dwarf binaries in coming years, with the hope of having a large enough sample to start looking at the systematics of these model tests. An additional improvement to our ability to test atmospheric and evolutionary models will come with the publication of parallax data for more brown dwarfs (which will allow precise luminosity measurements).

\section{References}

Allen, P. R. 2007, ApJ, 668, 492

Allers, K. N., Liu, M. C., Dupuy, T. J., \& Cushing, M. C. 2010, ApJ, 715, 561

Artigau, É., et al., 2011, ApJ, accepted. (arXiv:1107.0768)

Bate, M. R. 2009, MNRAS, 392, 590

Bergfors, C., et al., 2010, A\&SA, 520, A54

Biller, B., Allers, K., Liu, M., Close, L. M., \& Dupuy, T. 2011, ApJ, 730, 39

Burgasser, A. J., Bardalez-Gagliuffi, D. C., \& Gizis, J. E. 2011, AJ, 141, 70

Burgasser, A. J., Reid, I. N., Siegler, N., Close, L., Allen, P., Lowrance, P., \& Gizis, J. 2007, Protostars and Planets V, 427

Burgasser, A. J., Sitarski, B. N., Gelino, C. R., Logsdon, S. E., \& Perrin, M. D. 2011, ApJ, accepted. (arXiv:1107.1484)

Burrows, A., Heng, K., \& Nampaisarn, T. 2011, ApJ, 736, 47

Close, L. M., et al., 2007, ApJ, 660, 1492

Dupuy, T. J. \& Liu, M. C. 2011, ApJ, 733, 122

Dupuy, T. J., Liu, M. C., \& Ireland, M. J. 2009, ApJ, 692, 729

Duquennoy, A. \& Mayor, M. 1991, A\&A, 248, 485

Fischer, D. A. \& Marcy, G. W. 1992, ApJ, 396, 178

Gelino, C. R., et al., 2011, AJ, 142, 57

Joergens, V. 2008, A\&AA, 492, 545

Konopacky, Q. M., Ghez, A. M., Barman, T. S., Rice, E. L., Bailey, J. I., III, White, R. J., McLean, I. S., \& Duchêne, G. 2010, ApJ, 711, 1087

Kouwenhoven, M. B. N., Brown, A. G. A., Portegies Zwart, S. F., \& Kaper, L. 2007, A\& A, 474, 77 
Kraus, A. L. \& Hillenbrand, L. A. 2009, ApJ, 703, 1511

Liu, M. C., et al., 2011, ApJ, in press. (arXiv:1103.0014)

Liu, M. C., Dupuy, T. J., \& Ireland, M. J. 2008, ApJ, 689, 436

Liu, M. C., Dupuy, T. J., \& Leggett, S. K. 2010, ApJ, 722, 311

Luhman, K. L., Joergens, V., Lada, C., Muzerolle, J., Pascucci, I., \& White, R. 2007, Protostars and Planets V, 443

Mason, B. D., Hartkopf, W. I., Gies, D. R., Henry, T. J., \& Helsel, J. W. 2009, AJ, 137, 3358

Raghavan, D., et al., 2010, ApJS, 190, 1

Reipurth, B. \& Clarke, C. 2001, AJ, 122, 432

Stamatellos, D. \& Whitworth, A. P. 2009, MNRAS, 392, 413

\section{Discussion}

A. Burrows: There is not one set of predictions for the luminosity or effective temperature or radius of a brown dwarf at a given age. For a given age, there is a band of possible values of any three due to ambiguities in metallicities and, importantly, this fact needs to temper the zeal of observers when reaching conclusions concerning conflicts with theory. Moreover, ages for stars are not well-determined.

K. Allers: It is certainly true that observers need to use caution when comparing models. The example I presented of HD130948BC (Dupuy et al. 2009), is a brown dwarf binary with a dynamical mass measurement and with known age and metallicity from its G2V primary.

A. Burrows: How well can you determine the bolometric luminosity of your component brown dwarfs?

K. Allers: Distance determinations are usually the dominant source of error for brown dwarf binaries, as determining bolometric magnitudes by integrating optical to mid-IR photometry and spectroscopy is very straight-forward. For objects without parallax measurements, the uncertainty in $L_{b o l}$ can be as large as $50 \%$. The binaries I presented as tests for evolutionary models (HD130948BC and 2MASS J1534-2952) have some of the best distance determinations, yielding small uncertainties in $L_{b o l}(11 \%$ and $5 \%$, respectively).

P. ŠKODA: How many brown dwarfs are known in total? How are they discovered? Is there some systematic survey? And how are the BD binaries discovered? Systematic surveys?

K. Allers: To date, we know of $\sim 500$ brown dwarfs (spectroscopically confirmed). Most have been identified from large photometric surveys (2MASS, SDSS, DENIS). Of the $\sim 55$ known brown dwarf binaries, $\sim 95 \%$ have been discovered from high-resolution imaging surveys (HST or laser guide star) targeting nearby brown dwarfs.

R. Wilson: The 2010 version of the WD program gives distances that agree well with HIP parallax distances, so it can stand in for parallax in the few cases where bright and RV curves exist for eclipsing brown dwarfs. Older schemes for EB distance estimation could also do it, of course.

K. Allers: Unfortunately, we only know of one eclipsing brown dwarf binary system. 